\title{
Nanotechnology for the diagnosis and treatment of diseases
}

\author{
"An important advancement in the area of \\ drug delivery is the use of triggered response \\ nanoparticulate systems."
}

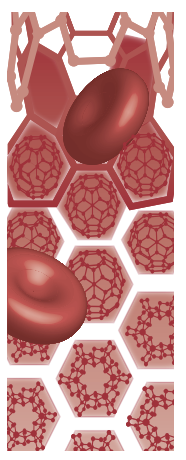

First submitted: 1 August 2016; Accepted for publication: 1 August 2016; Published online: 10 August 2016

This special issue of Nanomedicine was compiled after the 3rd International Conference on Nanotechnology in Medicine, held in Manchester, UK, in November 2015. It brought together international scientists and clinicians from multidisciplinary backgrounds and specialities to share a common interest in pushing the boundaries of our current understanding of the field of nanomedicine. This special issue focuses on key areas in the application of nanotechnology, with the aim of highlighting recent advances and developments to realize the full potential of nanomedicine.

Targeted drug delivery is a key application of nanotechnology in medicine that has gained a plethora of interest. While Feczko and colleagues illustrate the potential use of IFN- $\alpha$ drug-loaded nanocomposites as a potential tool for intravenous controlled release drug delivery [1], Quignard and colleagues formulated nanometric oil droplets for encapsulating solid nanoparticles as a way of modulating their internalization pathway and enhance intracellular delivery [2]. An important advancement in the area of drug delivery is the use of triggered response nanoparticulate systems. These include the use of a bioinspired mineralization process and superparamagnetic hybrid nanobeads. The work by Campodoni and colleagues provides a good example of performing such magnetic functionality [3]. They synthesized superparamagnetic hybrid nanobead hydroxyapatite composite material as a drug carrier system, with the ability of remote activation by a magnetic signal. This unique multifunctional drug delivery system is bioresorbable and biocompatible, holding promise for smart applications in nanomedicine, including active targeting and therapeutic purposes. An emergent technology in inhalation therapy is the use of targeted liposomes which has the potential of improving pulmonary delivery of drugs such as corticosteroids for patients with chronic asthma. Altube and colleagues synthesized $\mathrm{pH}$-sensitive archeosomes which are lipid vesicles extracted from archaebacterial that have the property of resistance to lipolytic enzymes [4]. These were loaded with dexamethasone as a model drug. The resultant nanoliposomes exhibited new pharmacodynamic properties with increased mechanical properties during nebulization, highlighting the potential of this novel concept.

Another key application of nanotechnology in medicine, enabled by advances in targeted drug delivery, is in therapeutics, in particular, cancer therapy. The review article by Karponis and colleagues illustrates the theranostic properties of magnetic nanoparticles as a promising candidate for the targeting and treatment of cancer [5]. This work was presented as a keynote lecture at the conference. Other nanoparticles, in particular, gold nanoparticles, also show significant potential as theranostic agents which integrate both diagnostic and therapeutic properties. Butterworth and colleagues illustrate clearly this concept in the simultaneous diagnosis and therapy of prostate cancer [6]. Using thiol stabilized gold nanoparticles, the authors were able to demonstrate enhanced
May Azzawi*,1, Alexander Seifalian ${ }^{2} \&$ Waqar Ahmed ${ }^{3}$ 'School of Healthcare Science, Faculty of Science \& Engineering, Manchester Metropolitan University, Manchester, UK ${ }^{2}$ Centre for Nanotechnology \& Regenerative Medicine, UCL Division of Surgery \& Interventional Science, University College London, London, UK ${ }^{3} \mathrm{~S}$ chool of Medicine, University of Central Lancashire, Preston, Lancashire, UK

*Author for correspondence: M.Azzawi@mmu.ac.uk 
CT image contrast and increased radiosensitivity of prostate tumors. This study presents one of the first examples of successful in vivo radiosensitization, combined by CT enhancement, in prostate cancer. Agrawal and colleagues describe the use of PEGylated chitosan nanoparticles as a delivery system for breast cancer therapy, to potentiate the anticancer activity of ormeloxifene [7]. The work was supported by in vivo pharmacokinetic and pharmacodynamic studies to demonstrate the significant improvement in therapeutic potential of the drug. The work by Afzal and colleagues highlights the potential of a folate-based strategy as a targeted delivery system for cancer therapy [8]. Using docetaxel as a model drug and tumor regression studies, they were able to demonstrate the superiority of folate as a targeting ligand over PEGylation and other labeling strategies.

\section{"Another emerging application of nanotechnology in medicine is its huge potential in diagnostics for the detection of biomarkers and pathogens.'}

Emerging applications of nanotechnology in medicine also include its huge potential in diagnostics for the detection of biomarkers and pathogens. These include the use of biosensors for the detection of analytes within solutions and body fluids. For example, Si Nanowire field effect transistor biosensors represent highly sensitive analytical devices that enable real-time detection of a range of molecular markers. Dmitrienko and colleagues propose a novel type of surface modification of the biosensor surface for highly specific detection of short RNAs for the early diagnosis of cancer and monitoring of disease progression [9]. Alatraktchi and colleagues demonstrate the application of nanotechnology in the rapid detection of pathogens [10]. The authors highlight the superiority of amperometry for the detection of pathogen markers and their superiority over standard spectrophotometric techniques as a direction toward point of care for the diagnosis of bacterial infections. Other applications of nanotechnology in combating infections include the development of a prototype catheter dressing which incorporates particles of chlorhexidine hexametaphosphate. These form a nanoparticulate salt at the catheter entry site to inhibit bacterial growth and reduce wound colonization, as demonstrated in the

\section{References}

1 Feczkó T, Fodor-Kardos A, Sivakumaran M, Shubhra Q. In-vitro interferon- $\alpha$ release from interferon- $\alpha$ and pegylated IFN- $\alpha$ loaded PLGA and PEG-PLGA nanoparticles. Nanomedicine (Lond.) 11(16), 2029-2034 (2016). work of Barbour and colleagues [11]. Such compounds may have great future potential as useful components in wound care materials to control infections.

While the potential use of nanoparticles in cancer therapy and diagnostics is clearly realized, Shukur and colleagues illustrate the dosage-dependent influence of nanoparticles on arterial function, which highlights the need to assess nanomaterial biocompatibility for maintaining vasodilation (hence organ perfusion) when administered intravenously and help identify strategies for their safe clinical administration [12]. Finally, the review article by Sivakumaran and colleagues highlights the paramount importance for accurate characterization of nanomaterials that are used in clinical diagnosis and therapeutics in order to realize the full potential of nanotechnology in medicine [13]. The authors explore a range of analytic techniques and measurement modalities for studying the physicochemical characteristics of nanomaterials and discuss the potential of a recent advancement in technology which combines therapeutics and diagnostic applications, namely Tunable Resistive Pulse Sensing. The tool enables a range of applications, including their use as a tool in the rapidly expanding field of exosome research and in the evaluation of interactions between nanomaterials and components of body fluids, which is key to evaluating their fate after systemic administration.

To conclude, nanomedicine is a rapidly expanding field, with continually emerging novel technologies. We look forward to the continued sharing of knowledge and expertise by a multidisciplinary team of scientists and clinicians to address key challenges and debates at the forthcoming 4th International Conference of Nanotechnology in Medicine, to be held in Warsaw, Poland, in November 2016.

Financial \& competing interests disclosure

The authors have no relevant affiliations or financial involvement with any organization or entity with a financial interest in or financial conflict with the subject matter or materials discussed in the manuscript. This includes employment, consultancies, honoraria, stock ownership or options, expert testimony, grants or patents received or pending, or royalties.

No writing assistance was utilized in the production of this manuscript.

2 Quignard S, Frébourg G, Chen Y, Fattaccioli J. Nanometric emulsions encapsulating solid particles as alternative carriers for intracellular delivery. Nanomedicine (Lond.) 11(16), 2059-2072 (2016).

3 Campodoni E, Adamiano A, Dozio S et al. Development of innovative hybrid and intrinsically magnetic nanobeads 
as drug delivery system. Nanomedicine (Lond.) 11(16), 2119-2130 (2016).

4 Altube MA, Selzer S, de Farias M, Portugal R, Morilla MJ, Romero EL. Surviving nebulization-induced stress: dexamethasone in $\mathrm{pH}$-sensitive archaeosomes. Nanomedicine (Lond.) 11(16), 2103-2117 (2016).

5 Karponis D, Azzawi M, Seifalian A. An arsenal of magnetic nanoparticles: perspectives in the treatment of cancer. Nanomedicine (Lond.) 11(16), 2131-2146 (2016).

6 Butterworth K, Nicol J, Ghita M et al. Preclincial evaluation of gold-DTDTPA nanoparticles as theranostic agents in prostate cancer radiotherapy. Nanomedicine (Lond.) 11(16), 2035-2047 (2016).

7 Agrawal S, Ahmad H, Dwived M et al. PEGylated chitosan nanoparticles potentiates repurposing of Ormeloxifene in breast cancer therapy. Nanomedicine (Lond.) 11(16), 2147-2169 (2016).

8 Afzal SM, Zubair Shareef M, Dinesh T, Veerabrahma K. Folate-PEG decorated docetaxel lipid nanoemulsion for improved antitumor activity. Nanomedicine (Lond.) 11(16), 2171-2184 (2016).
9 Dmitrienko EV, Naumova OV, Fomin BI et al. Surface modification of SOI-FET sensors for label-free and specific detection of short RNA analyte. Nanomedicine (Lond.) 11(16), 2073-2082 (2016).

10 Alatraktchi FA, Johansen HK, Molin S, Svendsen WE. Electrochemical sensing of biomarker for diagnostics of bacteria-specific infections. Nanomedicine (Lond.) 11(16), 2185-2195 (2016).

11 Barbour ME, Maddocks SE, Grady HJ et al. Chlorhexidine hexametaphosphate as a wound care material coating: antimicrobial efficacy, toxicity and effect on healing. Nanomedicine (Lond.) 11(16), 2049-2057 (2016).

12 Shukur A, Whitehead D, Seifalian A, Azzawi M. The influence of silica nanoparticles on small mesenteric arterial function. Nanomedicine (Lond.) 11(16), 2131-2146 (2016).

13 Sivakumaran M, Platt $M$. Tunable resistive pulse sensing: potential applications in nanomedicine. Nanomedicine (Lond.) 11(16), 2197-2214 (2016). 